\title{
Feeding difficulty is the dominant feature in 12 Chinese newborns with CHD7 pathogenic variants
}

Xiang Chen ${ }^{1 \dagger}$, Kai Yan ${ }^{1 \dagger}$, Yanyan Gao ${ }^{2}$, Huijun Wang ${ }^{3}$, Guoqiang Chen ${ }^{1}$, Bingbing Wu ${ }^{3}$, Qian Qin ${ }^{3^{*}}$, Lin Yang ${ }^{4^{*}}$ (D) and Wenhao Zhou ${ }^{1,3}$

\begin{abstract}
Background: CHARGE syndrome is characterized by coloboma, heart defects, choanal atresia, growth retardation, genitourinary malformation and ear abnormalities. The chromodomain helicase DNA-binding protein 7 (CHD7) gene is the major cause of CHARGE syndrome and is inherited in an autosomal dominant manner. Currently, the phenotype spectrum of CHARGE syndrome in neonatal population remain elusive. We aimed to investigate the phenotype spectrum of neonatal patients suspected to have CHARGE syndrome with pathogenic or likely pathogenic variants in the CHD7 gene.

Methods: We pooled next-generation sequencing data from the Neonatal Birth Defects Cohort (NBDC, ClinicalTrials. gov Identifier: NCT02551081) in Children's Hospital of Fudan University. The pathogenicity of novel variants was analyzed by bioinformatic and genetic analyses. Clinical information collection, Sanger sequencing and follow-up interviews were performed when possible. Cranial MRI of these patients was performed, the volumes of different regions of the brain were analyzed.
\end{abstract}

Results: A total of 12 unrelated patients in our cohort were found with CHD7 variants. Eight patients received a firm clinical diagnosis of CHARGE syndrome (Bergmann criteria, Blake criteria, Verloes criteria and Hale criteria). Three patients did not match any diagnostic criteria, and no patients matched the Verloes criteria. Phenotype spectrum analysis found that feeding difficulty was the dominant feature among this neonatal cohort. Six novel variants in the CHD7 gene (Glu2408*, Lys651*, c.5607 + 1G > T, Leu373Val, Lys2005Asnfs*37 and Gln1991*) were identified, expanding the variant database of the CHD7 gene. Cranial MRI analysis revealed significant volume loss in cingulate gyrus, occipital lobe, and cerebellum and volume gain in the left medial and inferior temporal gyri anterior white matter parts.

Conclusions: Based on a relatively unbiased neonatal cohort, we concluded that CHARGE syndrome and CHD7 gene variants should be suspected in newborns who have feeding difficulty, and one or more malformations.

Trial registration: Neonatal Birth Defects Cohort (NBDC, ClinicalTrials.gov identifier: NCT02551081).

Keywords: CHARGE syndrome, CHD7 gene, Variant, Feeding difficulty, Newborn

\footnotetext{
*Correspondence: qingqian89@outlook.com; yanglin_fudan@163.com

Xiang Chen and Kai Yan are Co-first author

${ }^{3}$ Shanghai Key Laboratory of Birth Defects, The Translational Medicine Center

of Children Development and Disease of Fudan University, Children's

Hospital of Fudan University, 399 Wanyuan Road, Shanghai 201102, China

${ }^{4}$ Departments of Endocrinology, Children's Hospital of Fudan University, 399

Wanyuan Road, Shanghai 201102, China

Full list of author information is available at the end of the article
}

(c) The Author(s). 2019 Open Access This article is distributed under the terms of the Creative Commons Attribution 4.0 International License (http://creativecommons.org/licenses/by/4.0/), which permits unrestricted use, distribution, and reproduction in any medium, provided you give appropriate credit to the original author(s) and the source, provide a link to the Creative Commons license, and indicate if changes were made. The Creative Commons Public Domain Dedication waiver (http://creativecommons.org/publicdomain/zero/1.0/) applies to the data made available in this article, unless otherwise stated. 


\section{Background}

CHARGE syndrome ([MIM:214800]) is a rare disease with a prevalence between 1:15,000 and 1:17,000. This syndrome is mainly characterized by coloboma, heart defects, choanal atresia, growth retardation, genitourinary malformation and ear abnormalities [1]. Minor manifestations include a distinctive face, esophagus malformation, hypothalamohypophyseal dysfunction and mental retardation [2, 3]. Clinical diagnostic criteria were proposed by Blake in 1998 [2] and revised by Verloes in 2005 [3]. The CHD7 gene is the only known gene [4] responsible for 90\% 95\% of typical CHARGE syndrome [1]. CHD is an acronym for chromodomian helicase DNA-binding proteins. CHD7 belongs to the CHD protein subfamily III, along with CHD5, CHD6, CHD8, CHD9 [5, 6]. CHD7 acts as an ATPdependent chromatin remodeler and transcriptional regulator preferring short linker DNA [7]. In mouse models, CHD7 participates in embryonic stem cell differentiation and regulates the transcription of tissue-specific genes [8]. The broad spectrum of CHARGE syndrome symptoms is related to the regulatory function of CHD7 in the multipotent migratory neural crest in the embryonic period [9]. Bergmann et al. reported that a large number of patients with a $C H D 7$ variant do not fulfill the clinical criteria of CHARGE syndrome. Mildly affected patients may be overlooked easily. So, in 2011, they emphasized that CHD7 analysis is helpful in the CHARGE syndrome diagnosis process and proposed a guideline for CHD7 analysis [10]. In 2016, new CHARGE syndrome diagnostic criteria was proposed by Hale with four major criteria and seven minor criteria [11]. Major standards include pathogenic variants in the CHD7 gene, coloboma, choanal atresia or cleft palate and ear (external, middle or inner) abnormalities. The inclusion criteria of CHARGE syndrome are two major criteria and any number of minor criteria. So genetic testing is increasingly important in CHARGE syndrome diagnostic process. In this study, we describe the phenotype spectrum of 12 neonatal patients carrying CHD7 variants. This is the largest sample size with a focus on CHARGE syndrome in the Chinses Han population.

\section{Methods}

\section{Inclusion criteria based on clinical features and genetic} analysis

We pooled data from the Neonatal Birth Defects Cohort (NBDC, ClinicalTrials.gov Identifier: NCT02551081) in Children's Hospital of Fudan University during 2016-01 to 2018-11. This data included 11,572 whole exome sequencing, 13,636 clinical exome sequencing and 1284 whole genome copy number microarray analyses. In this retrospective study, enrolled newborns met one of the following four criteria: 1) a previously established heterozygous pathogenic variant in the CHD7 gene, with data obtained from both the public database (HGMD and ClinVar) and internal database; 2) the same amino acid change as a previously established pathogenic variant; however, different nucleotide changes were accepted; 3) a novel (both public database and internal database) heterozygous null variant (nonsense, frameshift, canonical $+/-1$ or 2 splice sites, initiation codon, single or multiexon deletion) in CHD7 gene; and 4) a novel and de novo heterozygous variant with negative family history or inherited from the affected parents. All variants were classified according to ACMG guideline [12]. Patients were excluded if pathogenic copy number variants were identified using array-based comparative genomic hybridization (arry-CGH). Patients' information was obtained from clinical records. A clinical diagnosis of CHARGE syndrome was made based on the Bergmann criteria [10], Blake criteria [2], Verloes criteria [3] and Hale criteria [11].

\section{Next generation sequencing and sanger confirmation}

The criteria for genetic testing were approved by ethics committees of Children's Hospital, Fudan University (2014-107). Pretest counseling was performed by physicians. Informed consent was obtained from the patient's parents. High-throughput sequencing was performed according to standard protocols in Clinical Laboratory Improvement Amendments (CLIA: 99D2064856) compliant sequencing laboratory in Wuxi NEXTCODE (China). Sequences were generated using the Agilent ClearSeq Inherited Disease Kit, Illumina Cluster and SBS Kit. Next generation sequencing was performed on an Illumina HiSeq 2000/2500 platform. The detected variants were confirmed using PCR and PCR-amplified DNA products were subjected to direct automated sequencing (3500XL Genetic Analyzer, Applied Biosystems) according to the manufacturer's specifications. De novo variants were detected by parental evaluating via Sanger sequencing.

\section{Cranial MRI analysis}

Among the 12 affected neonates, 8 had T1- and T2weighted MRI head scans from the picture archiving and communication system (PACS) in Children's hospital of Fudan University. We matched these affected neonates with 92 control neonates of the same corrected gestational age and excluded 4 patients because of image registration failure caused by poor image quality. All digital imaging and communications in medicine (DICOMs) were concatenated into a NIfTi volume format by dcm2niix software [13]. Then, the brain images of the neonates were extracted through FSL BET based on the T2-weighted modality followed by N4 bias correction [14]. The skullstriped T2 images were normalized to the neonate-specific T2 weighted image template at 44 weeks corrected gestational age [15] using the SyN registration method in ANTS [16]. The T2 brain template included a parcellation 
atlas with 87 regions of interest (ROI). The volumes of each ROI in the brain were measured by the sum of the log relative Jacobian determinant from the nonlinear deformable field of the registration. The total brain volume was used as a nuisance variable for regression, and the $t$ statistic map was constructed from the linear regression as follows: ROI_volume $\sim \alpha$ variants $+\beta$ corrected_age $+\theta$ gender+ 1 .

\section{Results}

\section{Clinical diagnosis}

A total of 12 pathogenic/likely pathogenic variants in the $C H D 7$ gene were identified by next generation sequencing in 12 unrelated newborns. All enrolled patients were from nonconsanguineous couples from the Chinese Han population. All patients' family histories were negative. The clinical manifestations of each patient are shown in Table 1. The Bergmann criteria focuses on patients with suspected features of CHARGE syndrome with CHD7 analysis. According to Bergmann's criteria, Patient 2, 6, 7, 8, 9, 10, 11, and 12 matched the guidelines and needed further $\mathrm{CHD7}$ analysis. Patients 7, 8 and 11 matched two cardinal and one supportive criteria and need CHD7 analysis including MLPA. Patient 2, 6, 9 and 10 matched one cardinal criteria and one or more than one supportive criteria. According to Bergmann's criteria, the four patients needed a temporal bone CT first to detect typical semicircular canal abnormalities. The Blake criteria has four major criteria (coloboma, choanal atresia/stenosis, characteristic ear anomalies and cranial nerve dysfunction) and seven minor criteria. Definitive CHARGE syndrome should match 4 major or 3 major features and 3 minor features. Probable or possible CHARGE is defined as 1 or 2 major features and several minor features. Based on the Blake criteria, 8 patients $(2,3,6,7,8,10,11$ and 12$)$ were diagnosed with probable or possible CHARGE. The major criteria of Hale proposed in 2016 were coloboma, choanal atresia or cleft palate, ear abnormalities and pathogenic CHD7 variants. Patients matching two major criteria and any number of minor criteria are diagnosed with CHARGE syndrome. In this study, four patients (2, 3, 7 and 11) met Hale's criteria for CHARGE syndrome. Major Verlos criteria include coloboma, choanal atresia/ stenosis and hypoplasia/aplasia of the semicircular canals. Patient matching as least two major criteria can be considered to have CHARGE syndrome. As no patient in our cohort was reported with choanal atresia/ stenosis and abnormalities of the semicircular canals, no patient could be diagnosed with CHARGE syndrome based on the Verloes criteria. In this population, some typical features of CHARGE syndrome were observed, including aplasia/dysplasia of the semicircular canals, cleft lip/palate and choanal atresia.

\section{Variants of CHD7}

Twelve pathogenic/likely pathogenic variants in the $C H D 7$ gene were identified. We detected 6 reported pathogenic variants and 6 novel variants, including 4 frameshifts, 4 stop-gain, 2 splice-donor region, 1 intron variant and 1 missense variant (Table 2). Stop-gain and frameshift variants accounted for $67 \%$ of variants in this study. None of these variants are included in the gnomeAD database (http:// gnomad.broadinstitute.org/), the 1000 gnommeAD database (http://gnomad-old.broadinstitute.org/) or our internal database (1833 probands and 6893 families). Among all six novel variants, Glu2408* and Lys651* (NM_017780) were identified as de novo variants by Sanger sequencing. We mapped the 12 variants into the structure of the CHD7 protein (http://www.ebi.ac.uk/interpro/). Two variants were located in the chromodomain, two were located in the SANT domain, one in the ATP-binding domain belongs to the Helicase superfamily (Helicase $\mathrm{N}$ domain) and one was in the BRK domain. We did not detect CHD7 copy number variants in our study.

\section{Cranial MRI analysis results}

MRI analysis revealed significant volume loss in the cingulate gyrus, occipital lobe, and cerebellum and volume gain in the left medial and inferior temporal gyri anterior white matter parts among neonates with the CHD7 variant (Fig. 1). In addition, different subregions showed different levels of volume loss among these three regions (Table 3).

\section{Discussion}

The NGS test largely reduced the turnaround time of high-sequencing genetic tests; therefore, genetic diseases can be rapidly diagnosed in neonatal patients. To the best of our knowledge, this is the first study to investigate the phenotype spectrum of neonatal patients with likely pathogenic or pathogenic $\mathrm{CHD7}$ variants. According to the four reported diagnostic criteria of CHARGE syndrome, $0-67 \%$ of newborns received a clinical diagnosis. This may be because some typical features of CHARGE syndrome (aplasia/dysplasia of the semicircular canals, cleft lip/palate and choanal atresia) are not observed in this population, and some features present later in childhood (growth retardation and mental retardation) cannot be diagnosed in neonatal period. Proposed in 2016, pathogenic CHD7 variant status is now a major criterion in CHARGE syndrome diagnoses [11]. Criteria focusing on typical clinical phenotypes may exclude patients with a mild phenotype in early life.

Digestive and respiratory problems are the primary causes of postneonatal demise in CHARGE syndrome [17]. Complex digestive anomalies in patients with CHARGE syndrome are often highly prevalent at birth and require long-term management $[18,19]$. In this study, $67 \%(8 / 12)$ of patients had digestive system anomalies, 
Chen et al. BMC Medical Genetics

(2019) 20:93

Page 4 of 10

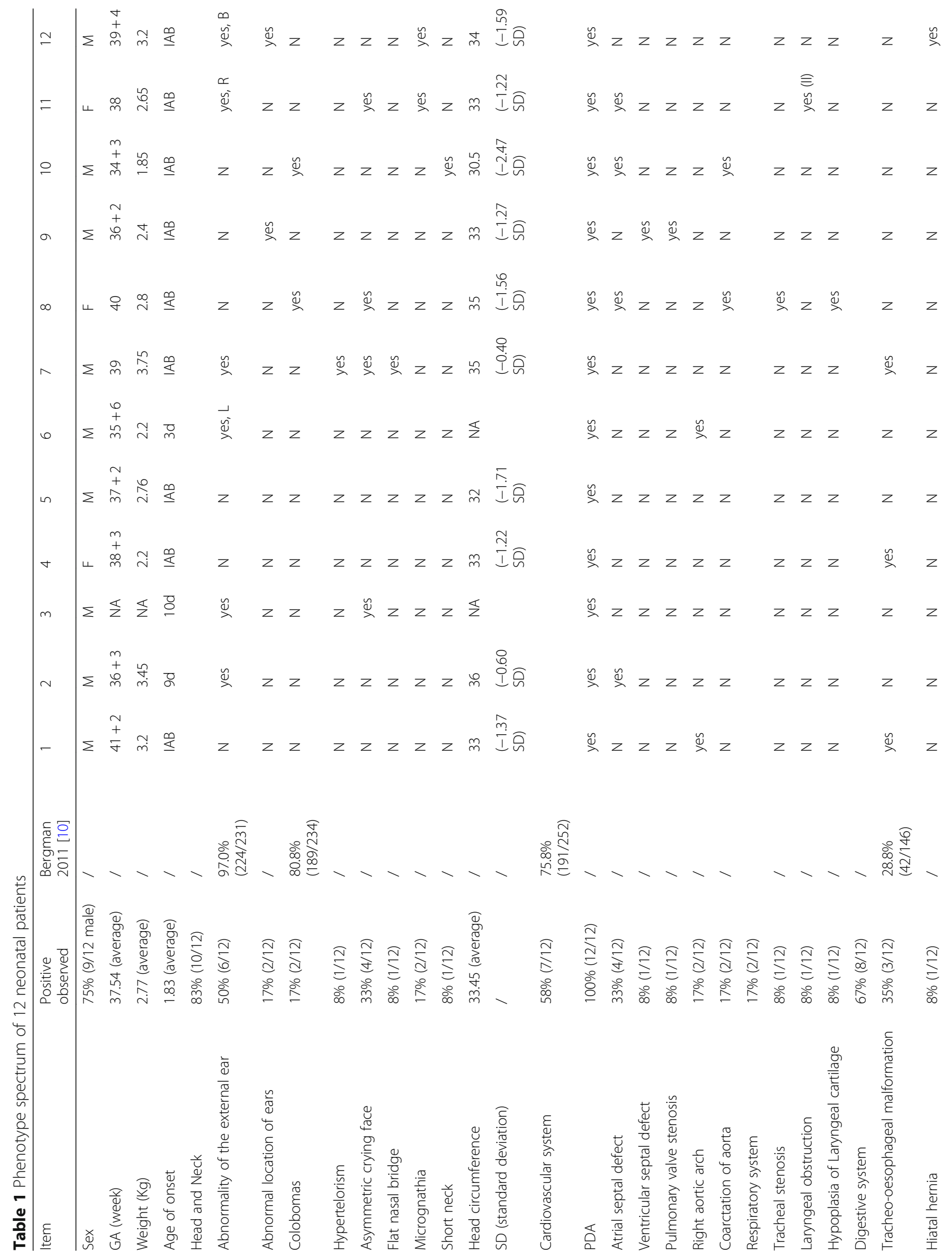


Chen et al. BMC Medical Genetics

(2019) 20:93

Page 5 of 10

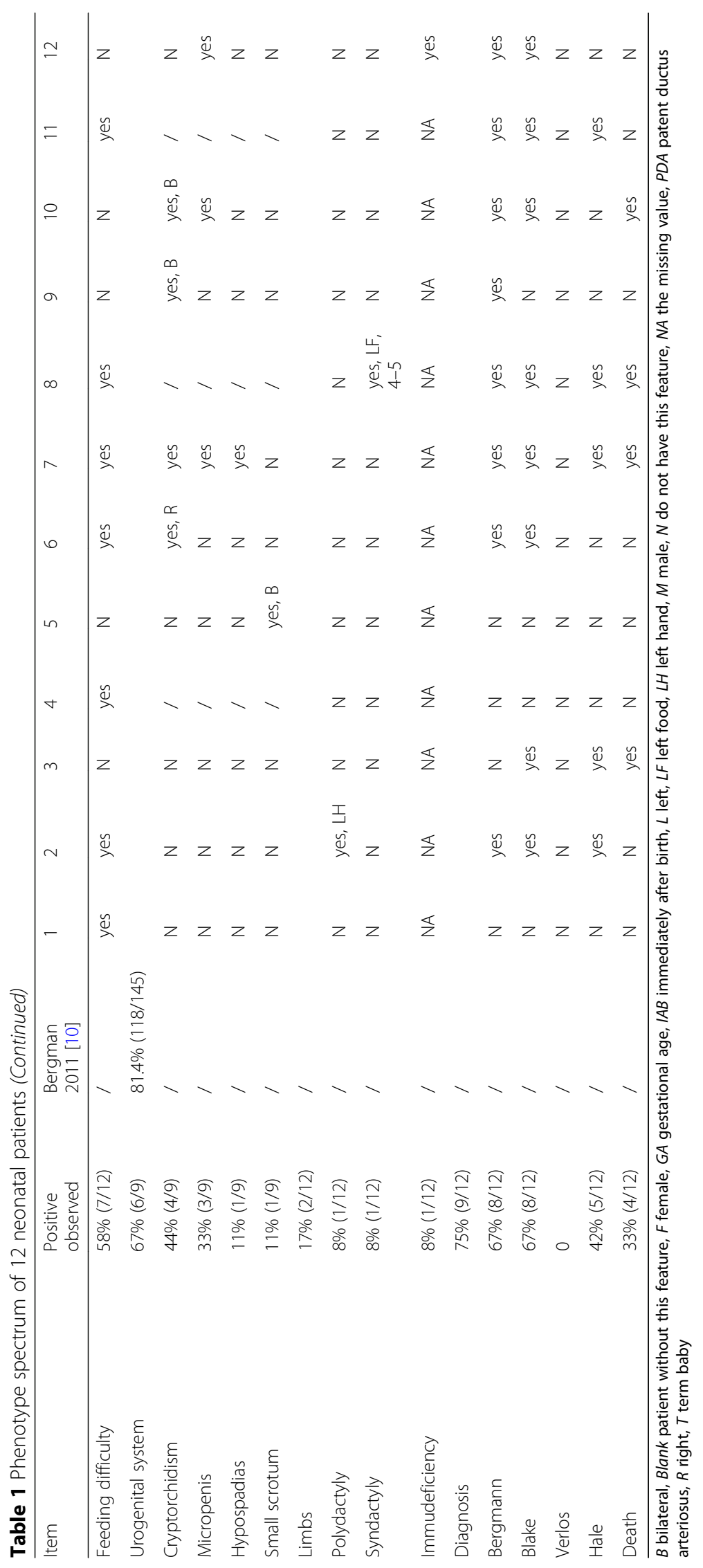




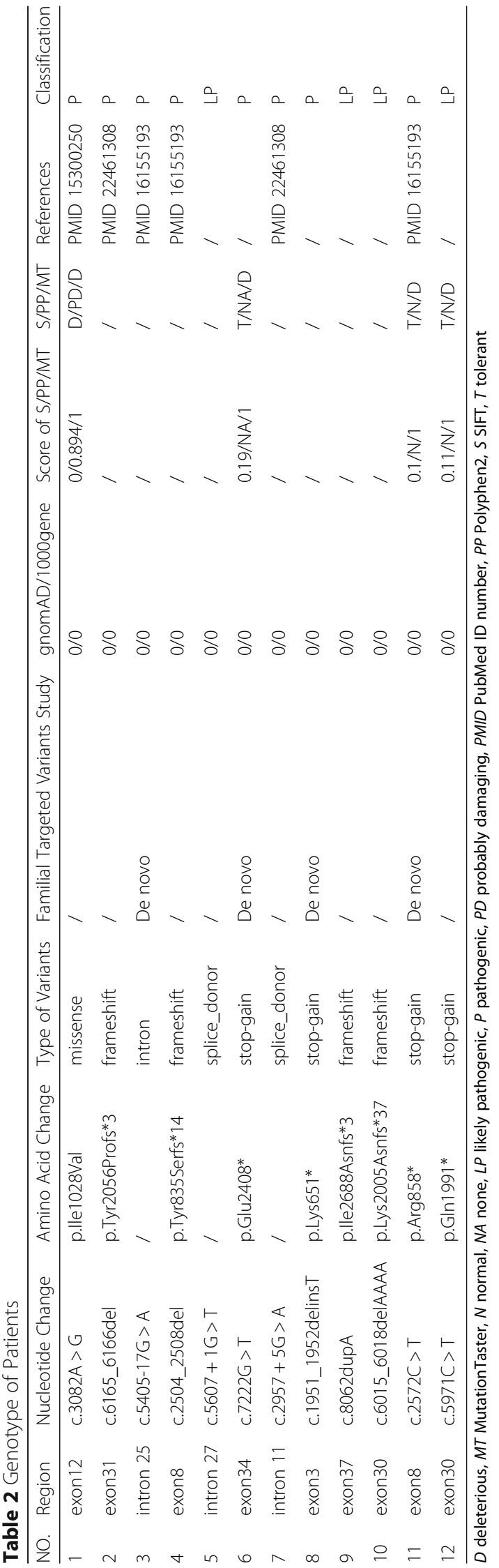




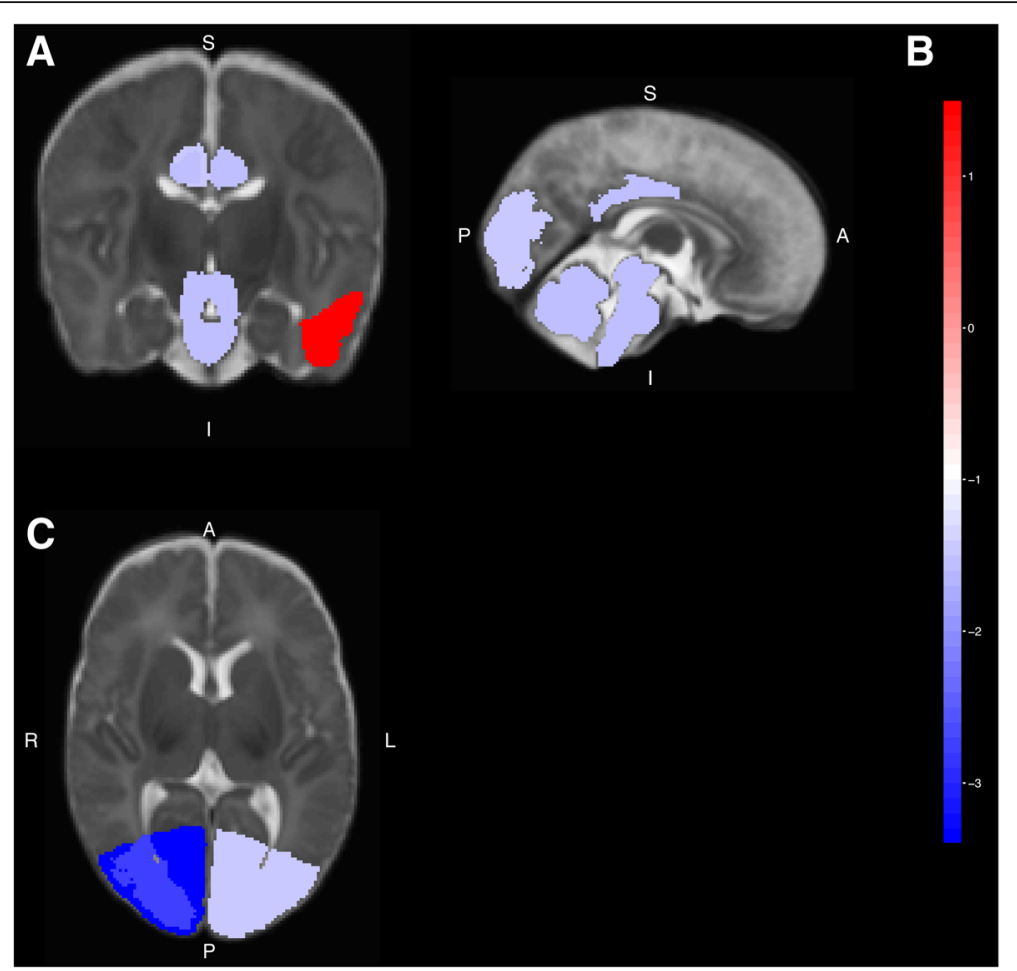

Fig. 1 Brain volume change of patients with pathogenic/likely pathogenic variants in CHD7. Red color means volume gain and blue color means volume loss $(p<0.05)$. (a) Coronal, (b) Sagittal, (c) Axial, R: Right; L: Left; A: Anterior; P: Posterior; I: Inferior; S: Superior

including feeding difficulty, tracheo-oesophageal malformation and hiatal hernia. Tracey Allen et al. [20] reported that tracheo-oesophageal malformation was regarded as a conditional finding in neonates and a major factor influencing morbidity. Tracheoesophageal fistula was previously reported as an uncommon feature among CHARGE syndrome patients with a prevalence between $8 \% \sim 18 \%$ [21]. However, Conny van Ravenswaaij-Arts [22] reported a higher prevalence $(28.8 \%, 42 / 146)$. In our study, 3 of 12 (25\%) patients had tracheo-oesophageal malformation.

Table 3 Volume loss regions of CHARGE syndrome patients

\begin{tabular}{lll}
\hline Region of Brain & Volume loss & $p$-value \\
\hline atlas50_17_Cerebellum left & -0.20947782 & 0.027094616 \\
atlas50_18_Cerebellum right & -0.229208438 & 0.016167437 \\
atlas50_22_Occipital lobe right & -0.341721802 & 0.000452598 \\
atlas50_23_Occipital lobe left & -0.210090999 & 0.037293534 \\
atlas50_34_Cingulate gyrus, posterior part right & -0.219671229 & 0.028380689 \\
atlas50_35_Cingulate gyrus, posterior part left & -0.220531078 & 0.029805553 \\
atlas7_6_Cerebellum + Brainstem & -0.20603902 & 0.026648778 \\
atlas87_22_Occipital lobe right GM & -0.370674616 & 0.000147048 \\
atlas87_23_Occipital lobe left GM & -0.230148083 & 0.022420291 \\
atlas87_27_Lateral occipitotemporal gyrus, gyrus fusiformis posterior part left GM & -0.192364134 & 0.0431107 \\
atlas87_34_Cingulate gyrus, posterior part right GM & -0.213154535 & 0.034225732 \\
atlas87_59_Medial and inferior temporal gyri anterior part left WM & 0.214177329 & 0.03865777 \\
atlas87_65_Occipital lobe right WM & -0.307703804 & 0.001591836 \\
atlas87_77_Cingulate gyrus, posterior part right WM & -0.217167864 & 0.030227307 \\
atlas87_78_Cingulate gyrus, posterior part left WM & -0.243852741 & 0.015847061 \\
atlas87_84_Extra-cranial background & -0.228192206 & 0.0277017 \\
\hline
\end{tabular}


Our results showed that feeding difficulty was the main feature among neonatal patients. Therefore, the possibility of CHARGE syndrome should be kept in mind when newborns suffer from feeding difficulty for a period.

Heart defects are predominant features in CHARGE syndrome patients and patients with pathogenic CHD7 variants [23]. Patent ductus arteriosus is more commonly seen in patients with $\mathrm{CHD} 7$ pathogenic variants than nonsyndromic heart malformations [24]. In our study, all patients had patent ductus arteriosus. As the patent ductus arteriosus can be closed within 3 months of age in most babies, the proportion of heart defects, including patent ductus arteriosus, may be overestimated in the neonatal population.

In our study, 4 neonates with CHARGE syndrome had an asymmetric crying face. To the best of our knowledge, this study first reported the asymmetric crying face in CHARGE syndrome. An asymmetric crying face is recognized in neonates as lower lip asymmetry present only with crying. The etiology includes facial nerve compression and faulty facial muscle/nerve development. An asymmetric crying face is often seen in 22q11.2 deletion syndrome patients; however, it has not been reported in CHARGE syndrome patients $[25,26]$. This could be because the importance of this symptom has not received enough attention.

Respiratory distress during feeding, regurgitation and persistent frothy salivation indicate a high risk of esophageal atresia or tracheoesophageal fistulae [27]. Treatment of feeding difficulty caused by esophageal atresia needs a multidisciplinary team to manage additional specific medical or physiological problems [28]. Although the survival of these patients is as high as $90 \%$, long-term complications are common, continuous and challenging [29]. Additionally, surviving patients diagnosed with CHARGE syndrome most likely suffer from development delays, especially in motor and language development [30].

For CHARGE syndrome patients with a $C H D 7$ pathogenic variant, the relationship between genotype and phenotype is unclear [10]. In this study, stop-gain, frameshift and splice variants were the main variants, which is in agreement with a previous study [31]. The patients in this study with the known pathogenic variant presented different phenotypes from the reported patients. All this indicates that CHARGE syndrome is a highly heterogeneous disease and $C H D 7$ gene analysis is important for comprehensive assessment.

MRI was used to detect cochlear abnormalities in CHARGE syndrome patients. Recent studies showed that $\mathrm{CHD} 7$ affects neurogenesis by activating neuron stem cells and progenitors [32]. Conditional genetic deficiency of Chd7 in mice led to abnormalities in corpus callosum and cerebellum [33, 34]. Christa M. de Geus et al. published a cranial imaging evaluation checklist for
CHARGE patients based on a literature review [35]. Cerebellum dysplasia was included as a cranial abnormality, but abnormalities of the cingulate gyrus and occipital lobe were not mentioned. Therefore, our study provides evidence to expand the abnormalities presented in the cranial imaging of CHARGE patients. According to previously studies, the cingulate gyrus processed and modulated gastrointestinal sensory signals [36], and the cerebellum involved in the regulation of feeding behavior [37]. These findings may explain why feeding difficulty is the dominant feature of neonatal CHARGE patients. The relationship between the occipital lobe, temporal lobe and feeding difficulty remains unknown. As only a few cranial MRI data of neonatal patients can be used for region division, only four patients were analyzed in this study. High-quality data collection from neonatal patients will be helpful for further study.

In this study, we reported 12 neonatal patients with CHD7 pathogenic/likely pathogenic variants. Six novel variants in the $C H D 7$ gene were identified, expanding the variant database of the $\mathrm{CHD} 7$ gene. Diagnostic criteria of CHARGE syndrome focusing on only typical clinical features may underestimate its neonatal incidence. CHARGE syndrome and CHD7 pathogenic variants should be suspected in newborns who have feeding difficulty and one or more malformations.

\section{Conclusions}

Our study found that diagnostic criteria of CHARGE syndrome focusing on only typical clinical features may underestimate neonatal incidence. Based on a relatively unbiased neonatal cohort, we concluded that CHARGE syndrome and $C H D 7$ gene variants should be suspected in newborns who have feeding difficulty, and one or more malformations.

\section{Additional file}

Additional file 1: Table S1. Filtered variations of 12 patients. (XLS 552 $\mathrm{kb})$

\section{Abbreviations}

CHD: chromodomain helicase DNA-binding; CLIA: Clinical Laboratory Improvement Amendments; DICOMs: digital imaging and communications in medicine; MLPA: multiplex ligation-dependent probe amplification; NBDC: Neonatal Birth Defects Cohort; NGS: next generation sequencing; PACS: picture archiving and communication system

\footnotetext{
Acknowledgements

We are very grateful to the patient families and thanks for their trust for our lab. Thanks the bioinformatic team members in our lab for data analysis. This work was funded by the National Natural Science Foundation of China (81720108018), the National Key Research and Development Program of China (2016YFC0905100), the Shanghai Municipal Commission of Health and Family Planning (GDEK201701), the Shanghai Shen Kang Hospital Development Center (SHDC12017110).
} 


\section{Funding}

This work was supported by grants from the National Natural Science Foundation of China (81720108018), the National Key Research and Development Program of China (2016YFC0905100), the Shanghai Municipal Commission of Health and Family Planning (GDEK201701), the Shanghai Shen Kang Hospital Development Center (SHDC12017110). They provided support for data collection and analysis.

\section{Availability of data and materials}

In this study, filtered variants' information of 12 patients were provided as Additional file 1: Table S1. Please contact corresponding author (Lin Yang) for more data.

\section{Authors' contributions}

CX performed data analyzing and wrote the draft of manuscript. YK collected MRI data and GYY collected clinical data. WHJ and WBB performed genetic diagnosis and consulting for patients in this cohort. CGQ performed clinical diagnosis. QQ performed MRI data analyzing and provided bioinformatic technique support. YL conceived this study and contributed to study design, results interpretation and critically edited the manuscript. ZWH was the Neonatal Cohort manager and contributed to study design and data analysis. All authors have read and approved the final manuscript.

\section{Ethics approval and consent to participate}

This study was approved by the ethics committees of Children's Hospital, Fudan University. The criteria for genetic testing were approved by ethics committees of Children's Hospital, Fudan University (2014-107). The informed consent was in the written from and was obtained from all participants.

\section{Consent for publication}

Written informed consents were obtained from the patients for publication of this study.

\section{Competing interests}

The authors declare that they have no competing interests.

\section{Publisher's Note}

Springer Nature remains neutral with regard to jurisdictional claims in published maps and institutional affiliations.

\section{Author details}

${ }^{1}$ Departments of Neonatology, Children's Hospital of Fudan University, Shanghai 201102, China. 'Ultrasonography Unit, Children's Hospital of Fudan University, Shanghai 201102, China. ${ }^{3}$ Shanghai Key Laboratory of Birth Defects, The Translational Medicine Center of Children Development and Disease of Fudan University, Children's Hospital of Fudan University, 399 Wanyuan Road, Shanghai 201102, China. ${ }^{4}$ Departments of Endocrinology, Children's Hospital of Fudan University, 399 Wanyuan Road, Shanghai 201102, China.

Received: 27 December 2018 Accepted: 23 April 2019

Published online: 30 May 2019

\section{References}

1. Hsu P, Ma A, Wilson M, Williams G, Curotta J, Munns CF, Mehr S. CHARGE syndrome: a review. J Paediatr Child Health. 2014;50:504-11.

2. Blake KD, Davenport SL, Hall BD, Hefner MA, Pagon RA, Williams MS, et al. CHARGE association: an update and review for the primary pediatrician. Clin Pediatr (Phila). 1998;37:159-73.

3. Verloes A. Updated diagnostic criteria for CHARGE syndrome: a proposal. Am J Med Genet A. 2005;133A:306-8.

4. Vissers LE, van Ravenswaaij CM, Admiraal R, Hurst JA, de Vries BB, Janssen $I M$, et al. Mutations in a new member of the chromodomain gene family cause CHARGE syndrome. Nat Genet. 2004;36:955-7.

5. Hall JA, Georgel PT. CHD proteins: a diverse family with strong ties. Biochem Cell Biol. 2007;85:463-76.

6. Marfella CG, Imbalzano AN. The Chd family of chromatin remodelers. Mutat Res. 2007;618:30-40.
7. Manning BJ, Yusufzai T. The ATP-dependent chromatin remodeling enzymes CHD6, CHD7, and CHD8 exhibit distinct nucleosome binding and remodeling activities. J Biol Chem. 2017;292:11927-36.

8. Schnetz MP, Bartels CF, Shastri K, Balasubramanian D, Zentner GE, Balaji R, et al. Genomic distribution of CHD7 on chromatin tracks H3K4 methylation patterns. Genome Res. 2009;19:590-601.

9. Bajpai R, Chen DA, Rada-Iglesias A, Zhang J, Xiong Y, Helms J, et al. CHD7 cooperates with PBAF to control multipotent neural crest formation. Nature. 2010;463:958-62.

10. Bergman JE, Janssen N, Hoefsloot LH, Jongmans MC, Hofstra RM, van Ravenswaaij-Arts CM. CHD7 mutations and CHARGE syndrome: the clinical implications of an expanding phenotype. J Med Genet. 2011;48:334-42.

11. Hale CL, Niederriter AN, Green GE, Martin DM. Atypical phenotypes associated with pathogenic CHD7 variants and a proposal for broadening CHARGE syndrome clinical diagnostic criteria. Am J Med Genet A. 2016; 170A:344-54.

12. Richards S, Aziz N, Bale S, Bick D, Das S, Gastier-Foster J, et al. Standards and guidelines for the interpretation of sequence variants: a joint consensus recommendation of the American College of Medical Genetics and Genomics and the Association for Molecular Pathology. Genet Med. 2015;17:405-24.

13. Li X, Morgan PS, Ashburner J, Smith J, Rorden C. The first step for neuroimaging data analysis: DICOM to NIfTI conversion. J Neurosci Methods. 2016;264:47-56.

14. Tustison NJ, Avants BB, Cook PA, Zheng Y, Egan A, Yushkevich PA, Gee JC. N4ITK: improved N3 bias correction. IEEE Trans Med Imaging. 2010;29:1310-20.

15. Oishi K, Mori S, Donohue PK, Ernst T, Anderson L, Buchthal S, et al. Multicontrast human neonatal brain atlas: application to normal neonate development analysis. Neuroimage. 2011;56:8-20.

16. Avants BB, Epstein CL, Grossman M, Gee JC. Symmetric diffeomorphic image registration with cross-correlation: evaluating automated labeling of elderly and neurodegenerative brain. Med Image Anal. 2008;12:26-41.

17. Bergman JE, Blake KD, Bakker MK, du Marchie SG, Free RH, Van RavenswaaijArts CM. Death in CHARGE syndrome after the neonatal period. Clin Genet. 2010;77:232-40

18. Hudson A, Macdonald M, Friedman JN, Blake K. CHARGE syndrome gastrointestinal involvement: from mouth to anus. Clin Genet. 2016.

19. Dobbelsteyn C, Peacocke SD, Blake K, Crist W, Rashid M. Feeding difficulties in children with CHARGE syndrome: prevalence, risk factors, and prognosis. Dysphagia. 2008;23:127-35.

20. Allen T. CHARGE syndrome: diagnosis and clinical management in the NICU Adv Neonatal Care 2012,12:336-342, 343-344.

21. Lalani SR, Safiullah AM, Fernbach SD, Harutyunyan KG, Thaller C, Peterson LE, et al. Spectrum of CHD7 mutations in 110 individuals with CHARGE syndrome and genotype-phenotype correlation. Am J Hum Genet. 2006;78:303-14.

22. van Ravenswaaij-Arts C, Martin DM. New insights and advances in CHARGE syndrome: diagnosis, etiologies, treatments, and research discoveries. Am J Med Genet C Semin Med Genet. 2017:175:397-406.

23. Corsten-Janssen N, Scambler PJ. Clinical and molecular effects of CHD7 in the heart. Am J Med Genet C Semin Med Genet. 2017:175:487-95.

24. Corsten-Janssen N, Kerstjens-Frederikse WS, du Marchie SG, Baardman ME, Bakker MK, Bergman JE, et al. The cardiac phenotype in patients with a CHD7 mutation. Circ Cardiovasc Genet. 2013;6:248-54.

25. Pasick C, McDonald-McGinn DM, Simbolon C, Low D, Zackai E, Jackson O. Asymmetric crying facies in the 22q11.2 deletion syndrome: implications for future screening. Clin Pediatr (Phila). 2013;52:1144-8.

26. Liang $X, H e B$. Congenital asymmetric crying facies syndrome: a case report. Medicine (Baltimore). 2018:97:e11403.

27. Aminde LN, Ebenye VN, Arrey WT, Takah NF, Awungafac G. Oesophageal atresia with tracheo-oesophageal fistula in a preterm neonate in Limbe, Cameroon: case report \& brief literature review. BMC Res Notes. 2014;7:692.

28. Ramsay M, Birnbaum R. Feeding difficulties in children with esophageal atresia: treatment by a multidisciplinary team. Dis Esophagus. 2013;26:410-2.

29. Smith N. Oesophageal atresia and tracheo-oesophageal fistula. Early Hum Dev. 2014:90:947-50.

30. Lalani SR, Hefner MA, Belmont JW, Davenport S. CHARGE Syndrome; 1993

31. Janssen N, Bergman JE, Swertz MA, Tranebjaerg L, Lodahl M, Schoots J, et al. Mutation update on the CHD7 gene involved in CHARGE syndrome. Hum Mutat. 2012;33:1149-60.

32. Feng W, Khan MA, Bellvis P, Zhu Z, Bernhardt O, Herold-Mende C, Liu HK. The chromatin remodeler CHD7 regulates adult neurogenesis via activation of SoxC transcription factors. Cell Stem Cell. 2013;13:62-72. 
33. He D, Marie C, Zhao C, Kim B, Wang J, Deng Y, et al. Chd7 cooperates with Sox 10 and regulates the onset of CNS myelination and remyelination. Nat Neurosci. 2016;19:678-89.

34. Feng W, Kawauchi D, Korkel-Qu H, Deng H, Serger E, Sieber L, et al. Chd7 is indispensable for mammalian brain development through activation of a neuronal differentiation programme. Nat Commun. 2017;8:14758.

35. de Geus CM, Free RH, Verbist BM, Sival DA, Blake KD, Meiners LC, van Ravenswaaij-Arts C. Guidelines in CHARGE syndrome and the missing link: cranial imaging. Am J Med Genet C Semin Med Genet. 2017;175:450-64.

36. Lawal A, Kern M, Sanjeevi A, Antonik S, Mepani R, Rittmann T, et al. Neurocognitive processing of esophageal central sensitization in the insula and cingulate gyrus. Am J Physiol Gastrointest Liver Physiol. 2008;294:G787-94.

37. Zhu JN, Wang JJ. The cerebellum in feeding control: possible function and mechanism. Cell Mol Neurobiol. 2008;28:469-78.

Ready to submit your research? Choose BMC and benefit from:

- fast, convenient online submission

- thorough peer review by experienced researchers in your field

- rapid publication on acceptance

- support for research data, including large and complex data types

- gold Open Access which fosters wider collaboration and increased citations

- maximum visibility for your research: over $100 \mathrm{M}$ website views per year

At $\mathrm{BMC}$, research is always in progress.

Learn more biomedcentral.com/submissions 